\title{
Riesgo de aerolización como limitante para el uso de cánulas de alto flujo en pacientes con insuficiencia respiratoria por Covid-19 de hospitales de recursos limitados
}

\section{Risk of aerolization as a limitation for the use of high flow cannulas in patients with respiratory insufficiency due to Covid-19 in limited resource hospitals}

Rodrigo Vergara-Tam, ${ }^{1,2, a}$, Ramón Ruesta-Berdejo, ${ }^{1,3, a}$, Fabricio CcamiBernal $^{1,4, a}$, Luis Castillo-Peña ${ }^{1,5, a}$, Miriam Arredondo-Nontol ${ }^{5,6,7, b}$

Señor editor:

Ha pasado más de un año desde la aparición de la COVID-19, se han desarrollado diferentes tipos de vacunas sin embargo a pesar de ello, debido a las demoras logísticas del proceso de vacunación y las mutaciones del virus, esta continúa propagándose. Ahora que está terminando la segunda ola, los sistemas de salud de hospitales públicos y privados de países subdesarrollados están colapsando nuevamente y agotando el uso de los ventiladores mecánicos invasivos en las Unidades de Cuidados Intensivos (UCI) ${ }^{(1,2)}$.

Una propuesta para este problema, sería el uso de las cánulas nasales de alto flujo (CNAF), las cuales ofrecen apoyo respiratorio a los pacientes con COVID-19 complicado por insuficiencia respiratoria hipoxémica aguda, disminuyendo la necesidad de ventilación mecánica invasiva ${ }^{(3)}$. Estos dispositivos se colocan en cada fosa nasal sin ocluirla y administran una mezcla de oxígeno y aire a un flujo superior a $1 \mathrm{~L}$ / min, regulable de acuerdo al efecto clínico ${ }^{(4)}$.

Sin embargo, la aceptación general para el uso de las CNAF es limitada debido a la controversia de estos dispositivos para crear aerosoles potencialmente infectantes ${ }^{(5)}$. Estudios preclínicos de simulación comparan el CNAF con dispositivos de bajo flujo, concluyendo que se producen una menor o igual cantidad de aerosoles ${ }^{(6)}$. Asimismo, no se han encontrado datos de contagio a personal de salud con el uso de este sistema. Adicionalmente, una revisión sistemática no rechaza el uso de las CNAF sopesando el beneficio de disminuir la necesidad de ventilación invasiva contra el riesgo desconocido de transmisión ${ }^{(3)}$.

Tomando en cuenta la situación de un país subdesarrollado frente al colapso sanitario, concluimos que, los riesgos potenciales del uso de las CNAF deben equilibrarse con los beneficios descritos previamente ${ }^{(5)}$. Por consiguiente, no se debería abandonar su utilización frente a otros dispositivos de oxígeno por los riesgos inciertos de transmisión del virus ${ }^{(7)}$ y se aconseja que el riesgo de dispersión por aerosoles de las CNAF se puede reducir mediante medidas sencillas como: uso de máscaras quirúrgicas, ajuste cuidadoso de las interfaces, sellado del circuito en pacientes asistidos y que el personal de salud utilice correctamente el equipo de protección personal ${ }^{(8)}$.

\section{REFERENCIAS BIBLIOGRÁFICAS}

1. Toledo-Leyva C. Peru, without ICU beds or oxygen in the middle of the second coronavirus wave [Internet]. 2021 [cited 2021 Feb 21]. Available from: https: / /bit.ly/3CpVXnh

2. Gestión. Private clinics without ICU beds available, fully occupied [Internet]. 2021 [cited 2021 Feb 21]. Available from: https: / / bit.ly/39jJ06T

3. Agarwal A, Basmaji J, Muttalib F, Granton D, Chaudhuri D, Chetan D, et al. High-flow nasal
FILIACIÓN

1. Unidad Generadora de Evidencias y Vigilancia Epidemiológica, Scientia Clinical and Epidemiological Research Institute, Trujillo,

2. Facultad de Medicina, Universidad Nacional de Trujillo, Trujillo, Perú.

Perú.

Piura, Perú.

Facultad de Medicina, Universidad Nacional de San Agustín de Arequipa, Arequipa, Perú

Facultad de Medicina, Universidad Nacional de Tumbes, Tumbes, Perú.

Unidad de Investigación Clínica, Scientia Clinical and Epidemiological Research Institute. Trujillo, Perú

Servicio de Pediatría, Hospital Carlos Alberto Cortez Jiménez, Tumbes, Perú

Estudiante de Medicina Humana

b Médico cirujano especialista en Pediatra.

ORCID

1. Rodrigo Vergara Tam /0000-0001-5629-3867

2. Ramón Ruesta Berdejo / 0000-0003-1380-3585

Ccami Bernal Fabricio / 0000-0003-3172-2113

Castillo Peña Luis/ 0000-0003-1752-9931

5. Arredondo-Nontol Miriam / $\underline{0000-0001-6269-7593}$

CORRESPONDENCIA

Arredondo-Nontol Miriam

Dirección: Scientia Clinical and Epidemiological Research Institute. Mz. G Lt. 22 Urb. Vista Hermosa - Trujillo - Perú.

Telefono: +51-988930781

EMAIL

rodfelian@gmail.com

CONFLICTOS DE INTERÉS

Los autores niegan conflictos de interés.

FINANCIAMIENTO

Autofinanciamiento.

REVISIÓN DE PARES

Recibido: 06/06/2021

Aceptado: 04/08/2021

COMO CITAR

Vergara-Tam, R., Ruesta-Berdejo, R., Ccami-Bernal, F., Castillo-Peña, L., \& Arredondo-Nontol, M. Riesgo de aerolización como limitante para el uso de canulas de alto flujo en pacientes con insuficiencia respiratoria por Covid-19 de hospitales de recursos limitados. Revista 14 ( https://doi.org/10.35434/rcmhnaaa.2021.14Sup1.1188

Esta obra está bajo una Licencia Creative Commons Atribución 4.0 Internacional.

Versión Impresa: ISSN: 2225-5109

Versión Electrónica: ISSN: 2227-4731

Versión Electrónica: ISSN: 2227-4731

Cross Ref. DOI: $10.35434 /$ rcmhnaaa
OJS: https://cmhnaaa.org.pe/ojs 
cannula for acute hypoxemic respiratory failure in patients with COVID-19: systematic reviews of effectiveness and its risks of aerosolization, dispersion, and infection transmission. Can J Anesth [Internet]. Springer International Publishing; 2020;67(9):1217-48. Available from: https: //bit.ly/3Ao7TFx

4. Wilkinson D, Andersen C, O'Donnell CPF, De Paoli AG MB. High flow nasal cannula for respiratory support in preterm infants. Cochrane Database Syst Rev. 2016;(2): CD006405. doi: 10.1002 14651858.CD006405.pub3.

5. Haymet A, Bassi GL, Fraser JF. Airborne spread of SARS-CoV-2 while using high-flow nasal cannula oxygen therapy: myth or reality? Intensive Care Med [Internet]. Springer Berlin Heidelberg; 2020;46(12):2248-51. Available from: https: / / bit.ly/39hz7BB
6. Gaeckle NT, Lee J, Park Y, Kreykes G, Evans MD, Hogan CJ. Aerosol generation from the respiratory tract with various modes of oxygen delivery. Am J Respir Crit Care Med. 2020;202(8):1115-24. doi: 10.1164 / rccm.202006-23090C.

7. Kluge S, Janssens U, Welte T, Weber-Carstens S, Marx G, Karagiannidis C. German recommendations for critically ill patients with COVID-19. Medizinische Klin - Intensivmed und Notfallmedizin. 2020; 115:111-4. doi: 10.1007 / s00063-020-00689-w.

8. Li J, Fink JB, Ehrmann S. High-flow nasal cannula for COVID-19 patients: Risk of bio-aerosol dispersion. Eur Respir J [Internet]. 2020;56(4):2-3. Available from: https: / / bit.ly/3ktNFot. 